\title{
COMPACT IMBEDDING THEOREMS FOR QUASIBOUNDED DOMAINS
}

\author{
BY \\ ROBERT A. ADAMS( $\left.{ }^{1}\right)$
}

1. Introduction. Let $G$ be an unbounded open set in Euclidean $n$-space $E_{n}$. In this paper we investigate (for a large class of such domains) the problem of determining for which values of $m, p, j$ and $r$ the Sobolev space imbedding

$$
W_{0}^{m, p}(G) \rightarrow W_{0}^{j, r}(G)
$$

is or is not compact. Provided $j<m$ continuous imbeddings of this type are known to exist for $p \leqq r \leqq n p(n-m p+j p)^{-1}$ if $n>m p-j p$ or for $p \leqq r<\infty$ if $n \leqq m p-j p$ (the Sobolev Imbedding Theorem, e.g. [5, Lemma 5]). If $G$ were bounded Kondrašov's compactness theorem [9] would yield the complete continuity of these imbeddings except in the extreme case $r=n p(n-m p+j p)^{-1}$. Such compactness theorems are useful for studying existence and spectral theory for partial differential operators on $G$.

In a sequence of recent papers the writer [1]-[4] and C. W. Clark [6], [7], [8] have studied such compactness problems for various unbounded domains. It is clear that the imbedding (1) cannot be compact if $G$ contains infinitely many disjoint congruent balls, for if a fixed $C^{\infty}$ function has support in one of these balls then the set of its translates with supports in the other balls is bounded in any space $W_{0}^{m, p}(G)$ but is not precompact in any such space. Thus a necessary condition for the compactness of imbedding (1) is that $G$ should be quasibounded, i.e. that dist $(x$, bdry $G) \rightarrow 0$ whenever $|x| \rightarrow \infty, x \in G$. In [1] the writer has shown that if $n>1$ then quasiboundedness is not sufficient for compactness.

The dimension of the boundary of $G$ is a critical factor in determining whether or not (1) is compact. If $G$ is quasibounded and bounded by smooth "reasonably unbroken" ( $n-1)$-dimensional manifolds then (1) is compact [3, Theorem 1] for any $m$ and $p$ and for the same values of $j$ and $r$ as in the case of bounded $G$. However if $G$ has discrete (0-dimensional) boundary then [2, Theorem 1] no such imbedding can be compact unless $m p>n$.

Our purpose in this paper is to study the compactness of imbedding (1) for quasibounded domains $G$ whose boundaries are comprised of smooth manifolds

Received by the editors June 6, 1969.

(1) Research sponsored by Air Force Office of Scientific Research, Office of Aerospace Research, United States Air Force, under AFOSR grant AFOSR-68-1531.

Copyright (C) 1970, American Mathematical Society 
of various dimensions. Roughly speaking our results are as follows. If $k$ is the smallest integer for which those boundary manifolds of $G$ having dimension not less than $n-k$ bound a quasibounded domain then no imbedding of type (1) can be compact when $m p<k$. On the other hand, if, in addition, the boundary manifolds are "reasonably unbroken" and if $m p>n+p-n p / k$ then (1) is compact for the same values of $j$ and $r$ as in the case of bounded $G$. Our results thus interpolate between the extreme cases mentioned above. We consider first domains $G$ with flat (planar) boundaries, establishing in $\$ 2$ a necessary condition for the compactness of (1) for such $G$, and in $\S 3$ a slightly stronger sufficient condition. If $m=1$ these conditions are equivalent for certain domains. In $\$ 4$ similar results are obtained for nonflatly-bounded domains $G$.

As usual, in this paper $W_{0}^{m, p}(G)$ denotes, for $p \geqq 1$ and $m=0,1,2, \ldots$, the Sobolev space obtained by completing with respect to the norm

$$
\|u\|_{m, p, G}=\left\{\sum_{j=0}^{m}|u|_{j, p, G}^{p}\right\}^{1 / p}
$$

the space $C_{0}^{\infty}(G)$ of all infinitely differentiable, complex functions having compact support in $G$ where

$$
|u|_{j, p, G}^{p}=\sum_{|\alpha|=j} \int_{G}\left|D^{\alpha} u(x)\right|^{p} d x
$$

$\alpha$ denotes an $n$-tuple of nonnegative integers $\left(\alpha_{1}, \ldots, \alpha_{n}\right) ;|\alpha|=\alpha_{1}+\cdots+\alpha_{n}$; $D^{\alpha}=D_{1}^{\alpha}{ }_{1} \cdots D_{n}^{\alpha} ; D_{j}=\partial / \partial x_{j}$. Note that $|u|_{0, p, G}=\|u\|_{0, p, G}$ is the norm of $u$ in $L^{p}(G)$. $W^{m, p}(G)$ represents the completion with respect to the norm (2) of the space of all infinitely differentiable functions on $G$ for which (2) is finite. Provided the boundary of $G$ satisfies certain mild regularity conditions [5, Lemma 5] the Sobolev Imbedding Theorem referred to above, and also (provided $G$ is bounded) the Kondrašov Compactness Theorem, remain valid for imbeddings of $W^{m, p}(G)$. No compactness theorems of this sort are yet known if $G$ is unbounded.

2. Flatly-bounded domains-noncompact imbeddings. Let $H$ be a $k$-dimensional plane $(0 \leqq k \leqq n-1)$ in $E_{n}$ and let $a$ be a point on $H$. With respect to a new system of rectangular coordinates $z$ in $E_{n}$ having origin at $a$ and obtained from the usual coordinates by an affine transformation, $H$ has equations $z_{1}=z_{2}=\cdots=z_{n-k}=0$, or more simply $r=0$ where $r=\sum_{i=1}^{n-k} z_{i}^{2}$. The coordinate $r$, together with $n-k-1$ angle coordinates collectively denoted $\sigma$ and the coordinates $z^{\prime}=\left(z_{n-k+1}, \ldots, z_{n}\right)$ form a system of cylindrical polar coordinates in $E_{n}$ with origin at $a$ and cylindrical axis $H$.

The $k$-tube $T_{\delta}(H)$ of radius $\delta$ and axis $H$ is the set $\left\{x \in E_{n}: \operatorname{dist}(x, H)=r<\delta\right\}$. By a tube function for the tube $T_{\delta}(H)$ we mean a $C^{\infty}$ function $\theta: E_{n} \rightarrow[0,1]$ whose value at $x$ depends only on $r=\operatorname{dist}(x, H)$ and which vanishes identically near $H$ and is identically unity outside $T_{\delta}(H)$. 
LEMMA 1. Let $H$ be a $k$-plane in $E_{n}$ and $a \in H$. Let $1 \leqq p<\infty$. If $u(x)=v(r)$ where $r=\operatorname{dist}(x, H)$ and $v \in C^{|\alpha|}((0, \infty))$ then for all $x \notin H$

$$
\left|D^{\alpha} u(x)\right|^{p} \leqq \text { const } \sum_{j=1}^{|\alpha|}\left|v^{(j)}(r)\right|^{p} r^{p j-p|\alpha|}
$$

where the constant depends only on $\alpha, p$ and $k$.

Proof. Since $z_{i}=\sum_{j=1}^{n} c_{i j}\left(x_{j}-a_{j}\right)$ and so $\partial / \partial x_{i}=\sum_{j=1}^{n} c_{j i} \partial / \partial z_{j}$ we may assume with no loss of generality that $H$ is a coordinate plane and $z=x$. We show that there exist homogeneous polynomials $P_{\alpha, j}(x)$ of degree $|\alpha|$ (possibly the zero polynomial) such that for $r>0$

$$
D^{\alpha} u(x)=\sum_{j=1}^{|\alpha|} P_{\alpha, j}(x) v^{(j)}(r) r^{j-2|\alpha|} .
$$

Since $\left|P_{\alpha, j}(x)\right| \leqq$ const $r^{|\alpha|}$ the conclusion of the lemma for $p=1$ follows at once. The result for general $p$ then follows from the well-known inequality

$$
\left|\sum_{j=1}^{N} A_{j}\right|^{p} \leqq \text { const } \sum_{j=1}^{N}\left|A_{j}\right|^{p}
$$

where the constant depends only on $p$ and $N$.

Note that $D^{\alpha} u(x)=0$ unless $\alpha_{n-k+1}=\cdots=\alpha_{n}=0$. If $1 \leqq i \leqq n-k$ then $D_{i} u(x)$ $=v^{\prime}(r) x_{i} / r$ which is of the required form. Assume (3) holds for all $\alpha$ with $|\alpha| \leqq m$. If $|\beta|=m+1$ then $D^{\beta}=D_{i} D^{\alpha}$ for some $i, \alpha$ where $|\alpha|=m$. Applying the induction hypothesis and the chain rule we obtain

$$
\begin{aligned}
D^{\beta} u(x)= & \sum_{j=1}^{m}\left\{D_{i} P_{\alpha, j}(x) v^{(j)}(r) r^{j-2 m}+P_{\alpha, j}(x) v^{(j+1)}(r) x_{i} r^{j-2 m-1}\right. \\
& \left.-(2 m-j) P_{\alpha, j}(x) v^{(j)}(r) x_{i} r^{j-2 m-2}\right\} \\
= & \sum_{j=1}^{m+1} P_{\beta, j}(x) v^{(j)}(r) r^{j-2(m+1)}
\end{aligned}
$$

where $P_{\beta, j}$ is given by

$$
\begin{aligned}
P_{\beta, 1}(x) & =r^{2} D_{i} P_{\alpha, 1}(x)-(2 m-1) x_{i} P_{\alpha, 1}(x), \\
P_{\beta, j}(x) & =r^{2} D_{i} P_{\alpha, j}(x)-(2 m-j) x_{i} P_{\alpha, j}(x)+x_{i} P_{\alpha, j-1}(x) \quad \text { if } 2 \leqq j \leqq m, \\
P_{\beta, m+1}(x) & =x_{i} P_{\alpha, m}(x) .
\end{aligned}
$$

Clearly $P_{\alpha, j}(x)$ is a polynomial of the desired type and the proof is complete.

Lemma 2. Let $\lambda$ be a positive integer and let $r=s^{\lambda}, s>0$. If $f \in C^{j}((0, \infty))$ and $1 \leqq p<\infty$ then

$$
\left|(d / d r)^{j} f\left(r^{1 / \lambda}\right)\right|^{p} \leqq \text { const } \sum_{i=1}^{j} \lambda^{-i p} s^{i p-j \lambda p}\left|f^{(i)}(s)\right|^{p}
$$

where the constant depends only on $j$ and $p$. 
Proof. Again the case of general $p$ follows from the special case $p=1$ via (4). For $p=1(5)$ is an immediate consequence of the formula

$$
(d / d r)^{j}=\lambda^{-j} \sum_{i=1}^{j} P_{j-i, j}(\lambda) s^{i-\lambda j}(d / d s)^{i}
$$

where $P_{i, j}$ is a polynomial of degree $i$ depending on $j$. We prove (6) by induction on $j$. Note that $d / d r=\lambda^{-1} s^{1-\lambda} d / d s$ which is of the required form. Assuming (6) we have

$$
\begin{aligned}
(d / d r)^{j+1} & =\lambda^{-j} \sum_{i=1}^{j} P_{j-i, j}(\lambda) \lambda^{-1} s^{1-\lambda} d / d s\left[s^{i-\lambda j}(d / d s)^{i}\right] \\
& =\lambda^{-(j+1)} \sum_{i=1}^{j} P_{j-i, j}(\lambda)\left\{s^{i+1-\lambda-\lambda j}(d / d s)^{i+1}+(i-\lambda j) s^{i-\lambda j-\lambda}(d / d s)^{i}\right\} \\
& =\lambda^{-(j+1)} \sum_{i=1}^{j+1} P_{j+1-i, j+1}(\lambda) s^{i-\lambda(j+1)}(d / d s)^{i}
\end{aligned}
$$

where the polynomials $P_{i, j+1}$ are given by

$$
\begin{aligned}
& P_{0, j+1}(\lambda)=P_{0, j}(\lambda), \\
& P_{i, j+1}(\lambda)=P_{i, j}(\lambda)+(j+1-i-\lambda j) P_{i-1, j}(\lambda) \quad \text { for } 1 \leqq i \leqq j-1, \\
& P_{j, j+1}(\lambda)=(1-\lambda j) P_{j-1, j}(\lambda),
\end{aligned}
$$

which are of the desired form.

Lemma 3. Let $T$ be a $k$-tube in $E_{n}$ with axis $H$ and radius $\delta \leqq 1$. Let $1 \leqq p<\infty$ and let $\lambda$ be a positive integer. Then there exists a tube function $\theta$ for $T$ satisfying for $|\alpha|>0$,

$$
\left|D^{\alpha} \theta(x)\right|^{p} \leqq \text { const } \lambda^{-p} s^{p-\lambda p|\alpha|}
$$

where $s^{\lambda}=r=\operatorname{dist}(x, H)$ and the constant depends only on $\alpha, n, p$ and $k$ and not on $\lambda$.

Proof. Let $f:[0, \infty) \rightarrow[0,1]$ be a fixed $C^{\infty}$ function such that $f(s)=0$ near $s=0$ and $f(s)=1$ for $s^{\lambda} \geqq \delta$. Define $\theta$ by $\theta(x)=v(r)=f(s)$. Clearly $\theta$ is a tube function for $T$. By Lemmas 1 and 2 we have

$$
\begin{aligned}
\left|D^{\alpha} \theta(x)\right|^{p} & \leqq \text { const } \sum_{j=1}^{|\alpha|} r^{p j-p|\alpha|}\left|v^{(j)}(r)\right|^{p} \\
& \leqq \text { const } \sum_{j=1}^{|\alpha|} \sum_{i=1}^{j} \lambda^{-i p} s^{i p-\lambda p|\alpha|}\left|f^{(i)}(s)\right|^{p} \\
& \leqq \text { const } \lambda^{-p} S^{p-\lambda p|\alpha|} .
\end{aligned}
$$

The final inequality follows because whenever $D^{\alpha} \theta(x) \neq 0, s<\delta^{1 / \lambda} \leqq 1$, and also $\left|f^{(i)}(s)\right| \leqq$ const for $1 \leqq i \leqq|\alpha|$.

In the following lemma we consider several $(n-k)$-tubes in $E_{n}$ simultaneously. Hence all the related quantities $\theta, r, \sigma, z^{\prime}, s, H$ carry subscripts ranging from 1 to $N$. 
LEMMA 4. Let $S$ be a bounded open set in $E_{n}$. Let $H_{1}, \ldots, H_{N}$ be a finite collection of $(n-k)$-planes which intersect $\bar{S}$. Let $m$ be a positive integer and let $\varepsilon>0$. If either $p>1$ and $m p \leqq k$ or $p=1$ and $m<k$ then there exists a function $\psi \in C^{\infty}\left(E_{n}\right)$ with the properties:

(i) $\psi(x)=0$ for $x$ near $\bigcup_{i=1}^{N} H_{i}$,

(ii) $0 \leqq \psi(x) \leqq 1$ for all $x$,

(iii) $\psi(x)=1$ for $x$ in $E_{n}-\bigcup_{i=1}^{N} T_{\delta}\left(H_{i}\right), \delta>0$,

(iv) $\left\|D^{\alpha} \psi\right\|_{0, p, s} \leqq \varepsilon$ for $0<|\alpha| \leqq m$.

Proof. We first consider the case that no two of the planes $H_{i}$ intersect in $\bar{S}$. It is then possible to choose $\delta \leqq 1$ small enough so that if $T_{i}=T_{\delta}\left(H_{i}\right)$ then $T_{i} \cap T_{j} \cap S$ is empty if $i \neq j$. By Lemma 3 there exist tube functions $\theta_{i}$ for $T_{i}$ satisfying

$$
\left|D^{\alpha} \theta_{i}(x)\right|^{p} \leqq \text { const } \lambda^{-p} s_{i}^{p-\lambda p|\alpha|}
$$

where $s_{i}^{\lambda}=r_{i}=\operatorname{dist}\left(x, H_{i}\right)$ and the constant is independent of $\lambda$ and $\alpha$ for $0<|\alpha| \leqq m$. Let $\psi(x)=\theta_{i}(x) \theta_{2}(x) \cdots \theta_{N}(x)$. Clearly $\psi$ satisfies (i)-(iii). Note that $D^{\alpha} \psi=0$ outside $\bigcup_{i=1}^{N} T_{i}$ and that $D^{\alpha} \psi(x)=D^{\alpha} \theta_{i}(x)$ in, $T_{i}$. We have

$$
\begin{aligned}
\left\|D^{\alpha} \psi\right\|_{0, p, S}^{p} & =\sum_{i=1}^{N} \int_{S \cap T_{i}}\left|D^{\alpha} \theta_{i}(x)\right|^{p} d x \\
& \leqq \text { const } \lambda^{-p} \sum_{i=1}^{N} \int_{S \cap T_{i}} s_{i}^{p-\lambda p|\alpha|} r_{i}^{k-1} d r_{i} d \sigma_{i} d z_{i}^{\prime} \\
& \leqq \text { const } \lambda^{1-p} \int_{0}^{1} s_{i}^{p-\lambda p|\alpha|+\lambda k-1} d s_{i} .
\end{aligned}
$$

The final constant depends on $\alpha, p, n, k, N$ and diam $S$ but not on $\lambda$. If $|\alpha| \leqq m$ and $m p \leqq k$ then $p-\lambda p|\alpha|+\lambda k>0$ and so

$$
\left\|D^{\alpha} \psi\right\|_{0, p, S}^{p} \leqq \text { const } \lambda^{1-p}(p+\lambda k-\lambda p|\alpha|)^{-1} .
$$

The expression on the right can be made arbitrarily small for sufficiently large $\lambda$ provided either $p>1$ or $m<k$. This establishes (iv).

The case of intersecting $H_{i}$ remains to be considered. Again pick $\delta<1$ small enough so that $T_{i} \cap T_{j} \cap S$ is empty whenever $H_{i} \cap H_{j} \cap \bar{S}$ is empty. Define $\theta_{i}$ and $\psi$ as above. The general Leibniz formula states

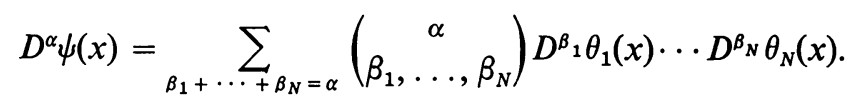

For estimates of $\left|D^{\alpha} \psi(x)\right|$ we may drop from terms in the Leibniz expression any factor $D^{\beta_{i}} \theta_{i}(x)$ for which $\beta_{i}=0$ because $\left|\theta_{i}(x)\right| \leqq 1$. For simplicity consider a term $D^{\beta_{1}} \theta_{1}(x) \cdots D^{\beta_{N}} \theta_{N}(x)$ where no $\beta_{i}$ is zero. Decompose $S$ into the union of $N$ subregions $S_{j}$ such that in $S_{j}$ we have $s_{j} \leqq s_{i}$ for $i \neq j$. We now obtain via Lemma 3 in the manner above, noting that whenever $D^{\beta_{i}} \theta_{i}(x) \neq 0$ then $s_{i}<\delta \leqq 1$ 


$$
\begin{aligned}
& \int_{S}\left|D^{\beta_{1}} \theta_{1}(x) \cdots D^{\beta_{N}} \theta_{N}(x)\right|^{p} d x=\sum_{j=1}^{N} \int_{S_{j}}\left|D^{\beta_{i}} \theta_{1}(x) \cdots D^{\beta_{N}} \theta_{N}(x)\right|^{p} d x \\
& \leqq \text { const } \lambda^{-N p} \sum_{j=1}^{N} \int_{S_{j}} s_{1}^{p-\lambda p\left|\beta_{1}\right| \cdots} s_{N}^{p-\lambda p\left|\beta_{N}\right|} d x \\
& \leqq \text { const } \lambda^{-N p} \sum_{j=1}^{N} \int_{S_{j}} s_{j}^{N p-\lambda p|\alpha|} d x \\
& \leqq \text { const } \lambda^{1-N p} \int_{0}^{1} s^{N p-\lambda p|\alpha|+\lambda k-1} d s \\
& \leqq \text { const } \lambda^{1-N p}(N p+\lambda k-\lambda p|\alpha|)^{-1} \text {. }
\end{aligned}
$$

Similar estimates can be found for all terms in (7) and (iv) follows once more by taking $\lambda$ sufficiently large.

Definition. Let $G$ be an open set in $E_{n}$. $G$ is called a regular domain if bdry $G$ $=\bigcup_{k=0}^{n-1} G_{k}$ where $G_{k}$ is the union of a locally finite collection of smooth manifolds of dimension $k$ in $E_{n}$. A regular domain $G$ whose boundary manifolds are all segments of planes of various dimensions will be called a regular flatly-bounded domain. An unbounded regular domain $G$ is called 0-quasibounded if it is quasibounded, i.e. if there exist at most finitely many disjoint congruent balls $Q$ in $G$, having any specified positive radius, which do not intersect the boundary of $G$. $G$ is called $k$-quasibounded $(1 \leqq k \leqq n-1)$ if there exist at most finitely many disjoint congruent balls $Q$ in $\bar{G}$, having any specified positive radius, such that $Q \cap$ bdry $G$ $\subset \bigcup_{i=0}^{k-1} G_{i}$; i.e. if $\operatorname{dist}\left(x, \bigcup_{i=k}^{n-1} G_{i}\right) \rightarrow 0$ as $|x| \rightarrow \infty, x \in G$. For $1 \leqq k \leqq n-1$ the condition of $k$-quasiboundedness is stronger than that of $(k-1)$-quasiboundedness.

THEOREM 1. Let $G$ be a regular, quasibounded, flatly-bounded domain in $E_{n}$. Let $k$ be the smallest integer $(1 \leqq k \leqq n)$ for which $G$ is $(n-k)$-quasibounded. If either $m p \leqq k$ and $p>1$ or $m<k$ and $p=1$ then no imbedding of the form

can be compact.

$$
W_{0}^{m . p}(G) \rightarrow W_{0}^{j, r}(G)
$$

Proof. For $2 \leqq k \leqq n$ since $G$ is not $(n-k+1)$-quasibounded there is a sequence of congruent open balls $\left\{Q_{i}\right\}_{i=1}^{\infty}$ in $\bar{G}$ such that $Q_{i} \cap$ bdry $G$ is contained in the union of finitely many $(n-k)$-planes. If $k=1$ balls $Q_{i}$ with this property exist trivially since $G$ is regular and flatly-bounded. Let $Q$ denote any one of these balls and let $H_{1}, \ldots, H_{N}$ be the corresponding $(n-k)$-planes. Let $\varphi \in C_{0}^{\infty}(Q)$ be a function for which

$$
\|\varphi\|_{0, r, Q}=2 C>0, \quad\|\varphi\|_{m, p, Q}=K<\infty .
$$

There exists a constant $M$ such that for all $x \in E_{n}$ and for all $\alpha$ with $0 \leqq|\alpha| \leqq m$, $\left|D^{\alpha} \varphi(x)\right| \leqq M$. Choose $\delta_{0}>0$ small enough so that the sum of the volumes of the intersections with $Q$ of the $(n-k)$-tubes $T_{\delta_{0}}\left(H_{i}\right), i=1, \ldots, N$, does not exceed $(C / M)^{r}$. Let

$$
\varepsilon=K\left[M \sum_{|\alpha| \leqq m} \sum_{\beta<\alpha}\left(\begin{array}{l}
\alpha \\
\beta
\end{array}\right)\right]^{-1} .
$$


By Lemma 4 there exists for some $\delta \leqq \delta_{0}$ a function $\psi \in C_{0}^{\infty}\left(E_{n}-\bigcup_{i=1}^{N} H_{i}\right)$ satisfying $0 \leqq \psi(x) \leqq 1, \psi(x)=1$ outside $\bigcup_{i=1}^{N} T_{\delta}\left(H_{i}\right)$ and $\left\|D^{\alpha} \psi\right\|_{0, p, Q} \leqq \varepsilon$ for $0<|\alpha| \leqq m$. Let $\gamma=\varphi \cdot \psi=\varphi-\varphi(1-\psi)$. Clearly $\gamma \in C_{0}^{\infty}(Q \cap G)$. Putting $T_{i}=T_{\delta}\left(H_{i}\right)$ we have

$$
\begin{aligned}
\|\gamma\|_{0, r, G} & \geqq\|\varphi\|_{0, r, Q}-\|\varphi\|_{0, r, Q \cap\left(T_{1} \cup \cdots \cup T_{N}\right)} \\
& \geqq 2 C-M\left[\operatorname{vol} Q \cap\left(T_{1} \cup \cdots \cup T_{N}\right)\right]^{1 / r} \geqq C .
\end{aligned}
$$

Moreover, for $0<|\alpha| \leqq m$

$$
\begin{gathered}
D^{\alpha} \gamma=\psi D^{\alpha} \varphi+\sum_{\beta<\alpha}\left(\begin{array}{l}
\alpha \\
\beta
\end{array}\right) D^{\beta} \varphi D^{\alpha-\beta} \psi, \\
\left\|D^{\alpha} \gamma\right\|_{0, p, Q} \leqq\left\|D^{\alpha} \varphi\right\|_{0, p, Q}+\sum_{\beta<\alpha}\left(\begin{array}{l}
\alpha \\
\beta
\end{array}\right) M \varepsilon \leqq 2 K .
\end{gathered}
$$

Thus if $K_{1}^{p}=\sum_{|\alpha| \leqq m} 1$ we have $\|\gamma\|_{m, p, G} \leqq 2 K K_{1}$.

Now let $\varphi_{i}, i=1,2, \ldots$ be a translate of $\varphi$ with support in $Q_{i}$ and let $\gamma_{i}$ be constructed from $\varphi_{i}$ as $\gamma$ from $\varphi$ above, so that

$$
\left\|\gamma_{i}\right\|_{0, r, G} \geqq C, \quad\left\|\gamma_{i}\right\|_{m, p, G} \leqq 2 K K_{1} .
$$

The sequence $\left\{\gamma_{i}\right\}$, though bounded in $W_{0}^{m, p}(G)$ has no subsequence converging in $L^{r}(G)$. In fact if $i \neq j$ then $\left\|\gamma_{i}-\gamma_{j}\right\|_{0, r, G} \geqq 2^{1 / r_{C}}$. Thus the imbedding

$$
W_{0}^{m, p}(G) \rightarrow W_{0}^{0, r}(G)=L^{r}(G)
$$

if it exists cannot be compact. Neither can the imbedding $W_{0}^{m, p}(G) \rightarrow W_{0}^{j, r}(G)$ for if this latter imbedding were compact then so would be the composition

$$
W_{0}^{m, p}(G) \rightarrow W_{0}^{j, r}(G) \rightarrow L^{r}(G) .
$$

3. Flatly-bounded domains-compact imbeddings. Let $H$ be a $k$-plane in $E_{n}$ and let $a \in H$. We denote by $T_{\delta, \rho}(H, a)$ the tube segment of radius $\delta$ and length $2 \rho$ having axis $H$ and centre $a$. Thus, if $P$ is the orthogonal projection operator on $H$ then

$$
T_{\delta, \rho}(H, a)=\left\{x \in E_{n}: \operatorname{dist}(x, H)<\delta, \operatorname{dist}(P x, a)<\rho\right\} .
$$

LEMmA 5. Let $H$ be an $(n-k)$-plane in $E_{n}(1 \leqq k \leqq n)$ and let $a \in H$. If either $p>k$ or $p=k=1$ then there exists a constant depending only on $p$ and $k$ such that for each $\delta, \rho>0$

$$
\|\gamma\|_{0, p, T} \leqq \text { const } \delta|\gamma|_{1, p, T}
$$

for all $\gamma \in C_{0}^{\infty}\left(E_{n}-H \cap T\right)$ where $T=T_{\delta, \rho}(H, a)$.

Proof. First consider the case $p>k$. Let $\left(r, \sigma, z^{\prime}\right)$ denote cylindrical polar coordinates in $E_{n}$ with origin at $a$ and cylindrical axis $H$. By Hölder's inequality for $\left(r, \sigma, z^{\prime}\right) \in T$ 


$$
\begin{aligned}
\left|\gamma\left(r, \sigma, z^{\prime}\right)\right|^{p} r^{k-1} & \leqq \delta^{k-1}\left|\int_{0}^{r} \frac{d}{d t} \gamma\left(t, \sigma, z^{\prime}\right) d t\right|^{p} \\
& \leqq \delta^{k-1} \int_{0}^{\delta}\left|D_{t} \gamma\left(t, \sigma, z^{\prime}\right)\right|^{p} t^{k-1} d t\left\{\int_{0}^{\delta} t^{-(k-1) /(p-1)} d t\right\}^{p-1} \\
& \leqq\left(\frac{p-1}{p-k}\right)^{p-1} \delta^{p-1} \int_{0}^{\delta}\left|D_{t} \gamma\left(t, \sigma, z^{\prime}\right)\right|^{p} t^{k-1} d t
\end{aligned}
$$

Integrating over $\Sigma$ and $Z$ the domains of the variables $\sigma$ and $z^{\prime}$ respectively in $T$ we obtain

$$
\begin{aligned}
\|\gamma\|_{0, p, T}^{p} & =\int_{\Sigma} d \sigma \int_{z} d z^{\prime} \int_{0}^{\delta}\left|\gamma\left(r, \sigma, z^{\prime}\right)\right|^{p} r^{k-1} d r \\
& \leqq\left(\frac{p-1}{p-k}\right)^{p-1} \delta^{p-1} \int_{0}^{\delta} d r \int_{\Sigma} d \sigma \int_{z} d z^{\prime} \int_{0}^{\delta}\left|D_{t} \gamma\left(t, \sigma, z^{\prime}\right)\right|^{p} t^{k-1} d t \\
& \leqq \text { const } \delta^{p}|\gamma|_{1, p, T}^{p} .
\end{aligned}
$$

For the special case $p=k=1$ we have

$$
\begin{aligned}
|\gamma(x)| & =\left|\gamma\left(r, z^{\prime}\right)\right| \leqq \int_{0}^{\delta}\left|D_{t} \gamma\left(t, z^{\prime}\right)\right| d t \\
\|\gamma\|_{0,1, T} & \leqq \int_{z} d z^{\prime} \int_{0}^{\delta} d r \int_{0}^{\delta}\left|D_{t} \gamma\left(t, z^{\prime}\right)\right| d t \leqq \delta|\gamma|_{1,1, T} .
\end{aligned}
$$

COROLlaRY. Under the conditions of the lemma, if $1 \leqq q \leqq p$ then there exists a constant depending only on $p, q, k, n$ such that for all $\delta>0$

$$
\|\gamma\|_{0, q, T} \leqq \text { const } \delta^{1+n / q-n / p}|\gamma|_{1, p, T}
$$

for all $\gamma \in C_{0}^{\infty}\left(E_{n}-H \cap T\right)$ where $T=T_{\delta, \delta}(H, a)$.

Proof. By Hölder's inequality and since vol $T=$ const $\delta^{n}$

$$
\begin{aligned}
\|\gamma\|_{0, q, T} & \leqq\|\gamma\|_{0, p, T}[\operatorname{vol} T]^{1 / q-1 / p} \\
& \leqq \text { const } \delta^{1+n / q-n / p}|\gamma|_{1, p, T} .
\end{aligned}
$$

Definition. Let $G$ be an unbounded, regular, flatly-bounded domain in $E_{n}$. We shall say that $G$ has the $k$-tube property if for every sufficiently large positive number $R$ there exists a positive number $\delta=\delta(R)$ with the properties:

(i) $\delta(R) \rightarrow 0$ as $R \rightarrow \infty$,

(ii) for each $x \in G_{R}=\{y \in G:|y|>R\}$ there exists a $k$-plane $H$ and a point $a \in H$ such that $x \in T_{\delta, \delta}(H, a)$ and $H \cap T_{2 \delta, 2 \delta}(H, a) \subset$ bdry $G$.

It is clear that if $G$ has the $k$-tube property then $G$ is $k$-quasibounded. Of course the converse is not true as the planar segments comprising the boundary of $G$ may have too many gaps to satisfy condition (ii). For domains $G$ whose boundaries consist only of whole planes the $k$-tube property is equivalent to $k$-quasibounded- 
ness. Other examples of domains with the $k$-tube property are not difficult to construct-for example Clark's "spiny urchin" [7] has the 1-tube property in $E_{2}$.

Lemma 6 (A VARIANT ON PoINCARÉ's INeQUality). Let $G$ be an unbounded, regular, flatly-bounded domain in $E_{n}$ having the $(n-k)$-tube property for some $k$ $(1 \leqq k \leqq n)$. If $1 \leqq r \leqq p$ where either $p>k$ or $p=k=1$ then there exists a constant depending only on $n, k, p$ and $r$ such that for all $u \in W_{0}^{1, p}(G)$ and all sufficiently large $R$

$$
\|u\|_{0, r, G_{R}} \leqq \text { const }[\delta(R)]^{1+n / r-n / p}|u|_{1, p, G} .
$$

Proof. Fix $R$ large enough so that $\delta=\delta(R)$ exists. If $\alpha$ is an $n$-tuple of integers (not necessarily nonnegative) let $Q_{\alpha}=\left\{x \in E_{n}: \alpha_{i} n^{-1 / 2} \delta \leqq x_{i} \leqq\left(\alpha_{i}+1\right) n^{-1 / 2} \delta\right\}$. Then $E_{n}=\bigcup_{\alpha} Q_{\alpha}$. If $x \in G_{R}$ then $x \in Q_{\alpha}$ for some $\alpha$ and there exists an $(n-k)$-plane $H$ and a point $a \in H$ such that $x \in T_{\delta, \delta}(H, a)=T$ and $H \cap T^{\prime} \subset$ bdry $G$ where $T^{\prime}$ $=T_{2 \delta, 2 \delta}(H, a)$. Clearly $Q_{\alpha} \subset T^{\prime}$. For any $\gamma \in C_{0}^{\infty}(G)$ since $\gamma$ vanishes near $H \cap T^{\prime}$ we have by the corollary of Lemma 5

$$
\begin{aligned}
\|\gamma\|_{0, r, Q_{\alpha} \cap G_{R}} & \leqq\|\gamma\|_{0, r, T^{\prime}} \\
& \leqq \text { const }\left.(2 \delta)^{1+n / r-n / p}\right|_{|\gamma|_{1, p, T^{\prime}}} \\
& \leqq \text { const } \delta^{1+n / r-n / p}|\gamma|_{1, p, Q_{\alpha}^{\prime}}
\end{aligned}
$$

where $Q_{\alpha}^{\prime}$ is the union of all the cubes $Q_{\beta}$ which intersect $T^{\prime}$. There is a number $N$ depending only on $n$ such that any $N+1$ of the sets $Q_{\alpha}^{\prime}$ have empty intersection. Summing the above inequality over all $\alpha$ for which $Q_{\alpha}$ meets $G_{R}$ we obtain

$$
\|\gamma\|_{0, r, G_{R}} \leqq \text { const } N \cdot \delta^{1+n / r-n / p}|\gamma|_{1, p, G}
$$

and this inequality extends by completion from $C_{0}^{\infty}(G)$ to $W_{0}^{1 . p}(G)$.

THEOREM 2. Let $G$ be an unbounded, regular, flatly-bounded domain in $E_{n}$ having the $(n-k)$-tube property $(1 \leqq k \leqq n)$. If either $p>k$ or $p=k=1$ then the imbedding $W_{0}^{m+1, p}(G) \rightarrow W_{0}^{m, r}(G)$ (exists and) is compact for $m=0,1,2, \ldots$ and $1 \leqq r<\infty$ if $p \geqq n$ or for $1 \leqq r<n p(n-p)^{-1}$ if $p<n$.

Proof. First consider the case $1 \leqq r \leqq p, m=0$. To prove that the imbedding $W_{0}^{1, p}(G) \rightarrow L^{r}(G)$ is compact we use the following compactness criterion for sets in $L^{r}(G)$ : a sequence $\left\{u_{i}\right\}_{i=1}^{\infty}$ which is bounded in $L^{r}(G)$ is precompact in $L^{r}(G)$ provided

(a) for every bounded $G^{\prime} \subset G$ the sequence $\left\{u_{i} \mid G^{\prime}\right\}$ is precompact in $L^{r}\left(G^{\prime}\right)$, and

(b) for each $\varepsilon>0$ there exists $R>0$ such that for all $i,\left\|u_{i}\right\|_{0, r, G_{R}}<\varepsilon$.

Lemma 6 and condition (i) of the $(n-k)$-tube property assures us that (b) is satisfied for any sequence $\left\{u_{i}\right\}$ bounded in $W_{0}^{1, p}(G)$. To establish (a) let $G^{\prime}$ be a bounded subset of $G$. Then for some $R, G^{\prime} \subset K_{R}=\left\{x \in E_{n}:|x| \leqq R\right\}$. Let $W^{1, p}(G, R)$ denote the completion with respect to the norm $\|\cdot\|_{1, p, G \cap K_{R}}$ of the space $C_{0}^{\infty}(G)$. The imbedding $W^{1, p}\left(K_{R}\right) \rightarrow L^{r}\left(K_{R}\right)$ is known to be compact (Kondrašov's Theorem) and since an element of $W^{1, p}(G, R)$ can be extended to be zero outside its support so as to belong to $W^{1, p}\left(K_{R}\right)$ it follows that $W^{1, p}(G, R)$ is compactly imbedded in 
$L^{r}\left(G \cap K_{R}\right)$. But $\left\{u_{i} \mid K_{R}\right\}$ is bounded in $W^{1, p}(G, R)$ and hence precompact in $L^{r}\left(G \cap K_{R}\right)$ whence $\left\{u_{i} \mid G^{\prime}\right\}$ is precompact in $L^{r}\left(G^{\prime}\right)$ as required.

By Sobolev's Imbedding Theorem $W_{0}^{1, p}(G)$ is continuously imbedded in $L^{q}(G)$ for any $q$ satisfying $p \leqq q<\infty$ if $p \geqq n$ or $p \leqq q \leqq n p(n-p)^{-1}$ if $p<n$. Select such a $q$ and a sequence $\left\{u_{i}\right\}$ bounded in $W_{0}^{1, p}(G)$ so that, say, $\left\|u_{i}\right\|_{0, q, G} \leqq C$. We may assume, passing to a subsequence if necessary, that $\left\{u_{i}\right\}$ converges in $L^{p}(G)$. By Hölder's Inequality if $p \leqq r<q$

$$
\begin{aligned}
\left\|u_{i}-u_{j}\right\|_{0, r, G} & \leqq\left\|u_{i}-u_{j}\right\|_{0, p, G}^{\lambda}\left\|u_{i}-u_{j}\right\|_{0, q, G}^{1-\lambda} \\
& \leqq(2 C)^{1-\lambda}\left\|u_{i}-u_{j}\right\|_{0, p, G}^{\lambda}
\end{aligned}
$$

where $\lambda=p(q-r) r^{-1}(q-p)^{-1}>0$. Hence $\left\{u_{i}\right\}$ converges in $L^{r}(G)$ and so the imbedding $W_{0}^{1, p}(G) \rightarrow L^{r}(G)$ is compact for $1 \leqq r<\infty$ if $p \geqq n$ and for $1 \leqq r<n p(n-p)^{-1}$ if $p<n$.

Finally, if $\left\{u_{\imath}\right\}$ is bounded in $W_{0}^{m+1, p}(G)$ then for any $\alpha$ with $0 \leqq|\alpha| \leqq m,\left\{D^{\alpha} u_{i}\right\}$ is bounded in $W_{0}^{1, p}(G)$ and so has a subsequence convergent to an element $v_{\alpha}$ of $L^{r}(G)$. In particular (for a suitable subsequence) $u_{i} \rightarrow v_{0}$ in $L^{r}(G)$ and so in the sense of distributions. Since $D^{\alpha} u_{i} \rightarrow v_{\alpha}$ in $L^{r}(G)$ and $D^{\alpha} u_{i} \rightarrow D^{\alpha} v_{0}$ in the sense of distributions it follows that $v_{\alpha}=D^{\alpha} v_{0}$ and $u_{i} \rightarrow v_{0}$ in $W_{0}^{m, r}(G)$. This completes the proof.

This theorem affords for imbeddings of the sort $W_{0}^{1, p}(G) \rightarrow L^{r}(G)$ on domains $G$ for which $(n-k)$-quasiboundedness is equivalent to the $(n-k)$-tube property, a complete converse to Theorem 1 . For imbeddings $W_{0}^{m, p}(G) \rightarrow L^{r}(G), m \geqq 2$, we do not fare quite so well.

THEOREM 3. Let $G$ be an unbounded, regular, flatly-bounded domain in $E_{n}$ having the $(n-k)$-tube property $(1 \leqq k \leqq n)$. Then the imbedding

$$
W_{0}^{m, p}(G) \rightarrow W_{0}^{j, r}(G), \quad 0 \leqq j<m,
$$

is compact in any of the following cases:

(i) $m=p=k=1$,

(ii) $m p>n+p-n p / k, p \leqq r<p^{*}$,

(iii) $m p>n+(j+1) p-n p / k, 1 \leqq r<p^{*}$, where $p^{*}=n p(n-m p+j p)^{-1}$ if $n>m p-j p$ and $p^{*}=\infty$ if $n \leqq m p-j p$.

Proof. The case $m=1$ has already been proved. If $m \geqq 2$ the imbedding $W_{0}^{m, p}(G) \rightarrow W_{0}^{1, q}(G)$ is continuous for $p \leqq q \leqq n p(n-m p+p)^{-1}$ if $n>m p-p$ and $p \leqq r<\infty$ if $n \leqq m p-p$. By Theorem 2 the imbedding $W_{0}^{1, q}(G) \rightarrow L^{p}(G)$ is compact provided $q>k$. Since $m p>n+p-n p / k$ is equivalent to $n p(n-m p+p)^{-1}>k$ such $q>k$ can always be chosen and so the composed imbedding $W_{0}^{m, p}(G) \rightarrow L^{p}(G)$ is compact.

By a standard interpolation theorem for Sobolev spaces [5, Lemma 6] there exists a constant $K$ such that for $0 \leqq j<m, p \leqq q<p^{*}$ we have

$$
\|u\|_{j, r, G} \leqq K\|u\|_{m, p, G}^{\lambda}\|u\|_{0, p, G}^{1}
$$


for all $u \in W_{0}^{m, p}(G)$ where $\lambda=(n r+j r p-n p)(m r p)^{-1}$. Note that $0 \leqq \lambda<1$ for all relevant values of $j, m, n, r$ and $p$. If $\left\{u_{i}\right\}_{i=1}^{\infty}$ is a bounded sequence in $W_{0}^{m, p}(G)$ then it has a subsequence again denoted $\left\{u_{i}\right\}$ which is convergent in $L^{p}(G)$. Since

$$
\begin{aligned}
\left\|u_{i}-u_{k}\right\|_{j, r, G} & \leqq K\left\|u_{i}-u_{k}\right\|_{m, p, G}^{\lambda}\left\|u_{i}-u_{k}\right\|_{0, p, G}^{1}-\lambda \\
& \leqq K\left[2 \sup \left\|u_{i}\right\|_{m, p, G}\right]^{\lambda}\left\|u_{i}-u_{k}\right\|_{0, p, G}^{1-\lambda},
\end{aligned}
$$

it follows that $\left\{u_{i}\right\}$ is a Cauchy sequence and hence convergent in $W_{0}^{j, r}(G)$ proving case (ii).

If $m p>n+(j+1) p-n p / k$ (and in particular if $j=0$ in case (ii)) we have by Sobolev's theorem and Theorem 2

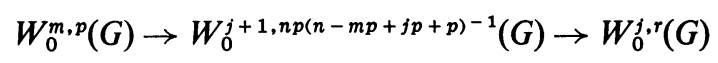

the second imbedding being compact since $n p(n-m p+j p+p)^{-1}>k$.

RemarK. The condition $m p>n+p-n p / k$ implies $m p>k$. The converse is, however, true for $m \geqq 2$ only if $k=n$ or $k \leqq p$. Thus even for domains $G$ for which $(n-k)$-quasiboundedness is equivalent to the $(n-k)$-tube property imbeddings of $W_{0}^{m, p}(G)$ corresponding to the cases $1<p<k<m p \leqq n+p-n p / k$ and $1=p<k \leqq m$ $\leqq n+1-n / k$ fail to be covered either by Theorem 1 or Theorem 3 .

4. Extensions to nonflatly-bounded domains. Let $G, G^{\prime}$ be open sets in $E_{n}$. A one-to-one transformation $M$ from $G$ onto $G^{\prime}$ is called an $m$-diffeomorphism of modulus $C$ if all the components of $M$ and $M^{-1}$ have continuous partial derivatives of all orders up to and including $m$, and these partials do not exceed $C$ in modulus.

Lemma 7. Let $G, G^{\prime}$ be open in $E_{n}$ and let $M$ be an m-diffeomorphism of modulus $C$ from $G$ onto $G^{\prime}$. Let

$$
A u(y)=u\left(M^{-1} y\right), \quad y \in G^{\prime}
$$

Then $A$ is a homeomorphism from $W^{m, p}(G)$ [respectively $W_{0}^{m, p}(G)$ ] onto $W^{m, p}\left(G^{\prime}\right)$ [resp. $\left.W_{0}^{m, p}\left(G^{\prime}\right)\right]$ and there exist constants $C_{1}$ and $C_{2}$ depending only on $n, p$ and $C$ and not on $G$ or $G^{\prime}$ such that for all $u \in W^{m, p}(G)$

$$
C_{1}\|u\|_{m, p, G} \leqq\|A u\|_{m, p, G^{\prime}} \leqq C_{2}\|u\|_{m, p, G}
$$

Proof. $A$ is a homeomorphism from $L^{p}(G)$ onto $L^{p}\left(G^{\prime}\right)$ for if $\partial M / \partial x$ represents the Jacobian determinant of $M$ then

$$
\begin{aligned}
\left\{\sup _{y \in G^{\prime}}\left|\frac{\partial M^{-1}(y)}{\partial y}\right|^{1 / p}\right\}^{-1}\|u\|_{0, p, G} & \leqq\|A u\|_{0, p, G^{\prime}} \\
& \leqq \sup _{x \in G}\left|\frac{\partial M(x)}{\partial x}\right|^{1 / p}\|u\|_{0, p, G} .
\end{aligned}
$$

By induction and formal applications of the chain rule it is easily verified that in the sense of distributions on $G^{\prime}$

$$
D^{\alpha}(A u)=\sum_{|\beta| \leqq|\alpha|} M_{\alpha \beta} A\left(D^{\beta} u\right)
$$


where $M_{\alpha \beta}$ is a polynomial of degree $|\beta|$ in the derivatives of the components of $M^{-1}$ involving derivatives of orders not exceeding $|\alpha|$. It follows that

$$
\begin{aligned}
\|A u\|_{m, p, C^{\prime}}^{p} & =\sum_{|\alpha| \leqq m}\left\|\sum_{|\beta| \leqq|\alpha|} M_{\alpha \beta} A\left(D^{\beta} u\right)\right\|_{0, p, G^{\prime}}^{p} \\
& \leqq \text { const } \sum_{|\beta| \leqq m}\left\|A\left(D^{\beta} u\right)\right\|_{0, p, G^{\prime}}^{p} \\
& \leqq \text { const }\|u\|_{m, p, G}^{p} .
\end{aligned}
$$

The reverse inequality follows in a similar manner.

LEMMA 8. Under the hypotheses of Lemma $7 W^{m, p}(G)$ is compactly imbedded in $W^{j, r}(G)$ if and only if $W^{m, p}\left(G^{\prime}\right)$ is compactly imbedded in $W^{j, r}\left(G^{\prime}\right)$. A similar statement holds for the spaces $W_{0}^{m, p}(G)$.

Proof. Suppose the imbedding $W^{m, p}(G) \rightarrow W^{j, r}(G)$ is compact. Let $\left\{u_{i}\right\}_{i=1}^{\infty}$ be a bounded sequence in $W^{m, p}\left(G^{\prime}\right)$. Then $\left\{A^{-1} u_{i}\right\}$ is bounded in $W^{m, p}(G)$ and so has a subsequence converging in $W^{j, r}(G)$. The corresponding subsequence of $\left\{u_{i}\right\}$ is convergent in $W^{j, r}\left(G^{\prime}\right)$ whence the imbedding $W^{m, p}\left(G^{\prime}\right) \rightarrow W^{j, r}\left(G^{\prime}\right)$ is compact. The other cases are proved similarly.

Of course Lemma 8 can be used to obtain immediately the conclusions of Theorems 1-3 for any domain $G$ which is $m$-diffeomorphic to an unbounded, regular, flatly-bounded domain $G^{\prime}$ satisfying the conditions of the particular theorem. As most quasibounded domains do not have this property we obtain generalizations of these theorems with localized hypotheses.

TheOREM 4. Let $G$ be a regular, unbounded domain in $E_{n}$. Let $k$ be the largest integer $(1 \leqq k \leqq n)$ for which for some constant $C$ there exist infinitely many mutually disjoint open sets $U$ in $\bar{G}$ each of which is m-diffeomorphic with modulus not greater than $C$ to the unit ball $B$ in $E_{n}$ in such a way that $U \cap$ bdry $G$ is mapped into a subset of the union of finitely many $(n-k)$-planes. (In particular $G$ is not $(n-k+1)$-quasibounded.) If either $m p \leqq k, p>1$ or $m<k, p=1$ then no imbedding of the form $W_{0}^{m, p}(G) \rightarrow W_{0}^{j, r}(G)$ can be compact.

Proof. Let $\left\{U_{i}\right\}_{i=1}^{\infty}$ be a sequence of mutually disjoint open sets in $\bar{G}$ for which there correspond $m$-diffeomorphisms $M_{i}: U_{i} \rightarrow B$ having modulus $\leqq C$ and such that $M_{i}\left(U_{i} \cap\right.$ bdry $\left.G\right) \subset B \cap P_{i}$ where $P_{i}$ is the union of finitely many $(n-k)$ planes. Let $\varphi \in C_{0}^{\infty}(B)$ be such that

$$
\|\varphi\|_{0, r, B}=C_{1}>0, \quad\|\varphi\|_{m, p, B}=K_{1}<\infty .
$$

By the method used in the proof of Theorem 1 we can construct functions $\gamma_{i} \in C_{0}^{\infty}\left(B-P_{i}\right)$ such that

$$
\left\|\gamma_{i}\right\|_{0, r, B} \geqq C_{2}>0, \quad\left\|\gamma_{i}\right\|_{m, p, B} \leqq K_{2}<\infty,
$$

the constants $C_{2}$ and $K_{2}$ being independent of $i$. Denoting by $A_{i}$ the operator for 
which $A_{i} u(y)=u\left(M_{i}^{-1} y\right), y \in B$ we have by Lemma 7 that there exist constants $C_{3}$ and $K_{3}$ again independent of $i$ such that

and

$$
\left\|A_{i}^{-1} \gamma_{i}\right\|_{0, r, G} \geqq C_{3}>0, \quad\left\|A_{i}^{-1} \gamma_{i}\right\|_{m, p, G} \leqq K_{3}<\infty
$$

$$
A_{i}^{-1} \gamma_{i} \in C_{0}^{\infty}\left(U_{i} \cap G\right) .
$$

The noncompactness of the imbedding $W_{0}^{m, p}(G) \rightarrow W_{0}^{j, r}(G)$ now follows as in Theorem 1.

As an analog of the compactness Theorems 2 and 3 we have

THEOREM 5. Let $G$ be an unbounded open set in $E_{n}$ with the property that there exist constants $C, R_{0}$ and $K$ such that for each $R \geqq R_{0}$ there exist positive numbers $d(R)$ and $\delta(R)$ with the following properties:

(i) $d(R)+\delta(R) \rightarrow 0$ as $R \rightarrow \infty$,

(ii) $d(R) / \delta(R) \leqq K, R \geqq R_{0}$,

(iii) for each $x \in G_{R}=\{x \in G:|x|>R\}$ the ball $B_{\delta}(x)$ of radius $\delta(R)$ and center $x$ can be mapped by a 1-diffeomorphism $M$ of modulus $\leqq C$ onto a set $S$ in $E_{n}$ such that for some $(n-k)$-plane $H(1 \leqq k \leqq n)$ and some point $a \in H$ we have $S \subset T_{d(R), d(R)}(H, a)$ and $H \cap T_{d(R), d(R)}(H, a) \subset M\left(\right.$ bdry $\left.G \cap B_{\delta}(x)\right)$.

Then the imbedding $W_{0}^{m, p}(G) \rightarrow W_{0}^{j, r}(G), 0 \leqq j<m$ is compact in any of the following cases:

(a) $m=p=k=1$,

(b) $m p>n+p-n p / k, p \leqq r<p^{*}$,

(c) $m p>n+(j+1) p-n p / k, 1 \leqq r<p^{*}$,

where $p^{*}=n p(n-m p+j p)^{-1}$ if $n>m p-j p$ and $p^{*}=\infty$ if $n \leqq m p-j p$.

Proof. The conclusion is the same as that of Theorem 3 and the proof is identical if we reprove Lemma 6 (Poincaré's inequality) under the conditions of this theorem. Thus, let $p>k$ or $p=k=1$ and let $1 \leqq r \leqq p$. Fix $R \geqq R_{0}$ and let $d=d(R)$ and $\delta=\delta(R)$. Define the cubes $Q_{\alpha}$ as in the proof of Lemma 6. If $x \in G_{R}$ then for some $\alpha, x \in Q_{\alpha}$ $\subset B_{\delta}(x)$. There exists a 1-diffeomorphism $M$ of $B=B_{\delta}(x)$ onto $S \subset E_{n}$ having modulus $\leqq C$ and there exists an $(n-k)$-plane $H$ and a point $a \in H$ such that $S \subset T=$ $T_{d, d}(H, a)$ and $H \cap T \subset M(B \cap$ bdry $G)$. For any $\gamma \in C_{0}^{\infty}(G)$ we have that $A \gamma$ (defined by $A \gamma(y)=\gamma\left(M^{-1} y\right), y \in S$ ) vanishes near $H \cap T$. Thus by the corollary of Lemma 5, Lemma 7 and the fact that $d \leqq K \delta$

$$
\begin{aligned}
\|\gamma\|_{0, r, Q_{\alpha} \cap G_{R}} & \leqq\|\gamma\|_{0, r, B} \leqq \text { const }\|A \gamma\|_{0, r, S} \\
& \leqq \text { const } d^{1+n / r-n / p}\|A \gamma\|_{1, p, T} \\
& \leqq \text { const } \delta^{1+n / r-n / p}\|\gamma\|_{1, p, M^{-1}(T)} \\
& \leqq \text { const } \delta^{1+n / r-n / p}\|\gamma\|_{1, p, Q_{\alpha}^{\prime}}
\end{aligned}
$$

where $Q_{\alpha}^{\prime}$ is the union of all the cubes $Q_{\beta}$ which intersect $M^{-1}(T)$. Since the modulus of $M$ is bounded, $M^{-1}$ is Lipschitzian and there exists a constant $\lambda$ such that $M^{-1}(T) \subset B_{\lambda d}(x) \subset B_{\lambda K \delta}(x)$. Thus there is a constant $N$ independent of $R$ and $x$ 
such that any $N+1$ of the sets $Q_{\alpha}^{\prime}$ have empty intersection. Summing the above inequality over those $\alpha$ for which $Q_{\alpha}$ meets $G_{R}$ we obtain, as in Lemma 6 , the required form of Poincaré's inequality.

5. An application to differential operators. Let $L$ be a linear partial differential operator of order $2 m$ in $G$ given by

$$
L u(x)=\sum_{|\alpha| \leqq 2 m} a_{\alpha}(x) D^{\alpha} u(x)
$$

with coefficients $a_{\alpha}$ infinitely differentiable, bounded, complex functions on $G$. Suppose $L$ is such that it satisfies the boundedness condition

$$
\left|\int_{G} L \varphi(x) \overline{\psi(x)} d x\right| \leqq c_{0}\|\varphi\|_{m, 2, G}\|\psi\|_{m, 2, G}
$$

for all $\varphi, \psi \in C_{0}^{\infty}(G)$, and also Garding's inequality

$$
\operatorname{Re} \int_{G} L \varphi(x) \overline{\varphi(x)} d x \geqq c_{1}\|\varphi\|_{m, 2, G}^{2}-c_{2}\|\varphi\|_{0,2, G}^{2}
$$

for all $\varphi \in C_{0}^{\infty}(G)$, where $c_{0}, c_{1}>0$ and $c_{2}$ are constants. The realization of $L$ in $L^{2}(G)$ corresponding to null Dirichlet boundary data is an operator $T$ in $L^{2}(G)$ defined by

$$
\begin{aligned}
\operatorname{Dom}(T) & =W_{0}^{m, 2}(G) \cap\left\{f \in L^{2}(G): L f \in L^{2}(G)\right\} \\
T f & =L f, \quad f \in \operatorname{Dom}(T) .
\end{aligned}
$$

THEOREM 6. If $G$ is open in $E_{n}$ and satisfies the conditions of either Theorem 3 or Theorem 5 with $2 m>n+2-2 n / k$ then $T$ as defined above is a closed linear operator in $L^{2}(G)$; the spectrum $\sigma(T)$ is discrete and has no finite limit points; for $\lambda \notin \sigma(T)$ the resolvent operator $R_{\lambda}(T)=(\lambda I-T)^{-1}$ is completely continuous.

The proof is identical to that of the standard theorem of this type. A sketch can be found in [6].

\section{REFERENCES}

1. R. A. Adams, Compact Sobolev imbeddings for unbounded domains, Pacific J. Math. (to appear).

2. - Compact Sobolev imbeddings for unbounded domains with discrete boundaries, J. Math. Anal. Appl. 24 (1968), 390-394.

3. - The Rellich-Kondrachov theorem for unbounded domains, Arch. Rational Mech. Anal. 29 (1968), 370-394. MR 37 \#3349.

4. - Compact Sobolev imbeddings for "pepper sets", J. Math. Anal. Appl. 27 (1969), 405-408.

5. F. E. Browder, On the spectral theory of elliptic differential operators. I, Math. Ann. 142 (1960/61), 22-130. MR 35 \#804.

6. C. W. Clark, An embedding theorem for function spaces, Pacific J. Math. 19 (1966), 243-251. MR 34 \#4946. 
7. C. W. Clark, Rellich's embedding theorem for a "spiny urchin", Canad. Math. Bull. 10 (1967), 731-734. MR 37 \#1989.

8. - Some embedding theorems for Sobolev spaces, (to appear).

9. V. I. Kondrašov, On certain properties of functions in the space $L_{p}$, Dokl. Akad. Nauk SSSR 48 (1945), 563-566.

UNIVERSITY OF BRITISH COLUMBIA,

VANCOUVER, B.C., CANADA 